\title{
A Novel Mathematical Analysis for Electrical Specifications of Step-up Converter
}

\author{
Davood GHADERI, Gökay BAYRAK
}

\begin{abstract}
This study presents a unique comprehensive mathematical model for both transient and steady states of the step-up power converter in order to structure physical aspects evaluations. The main disadvantage of different existence mathematical models such as impedance, small signal analysis and steady space average models is that they use numerical analysis methods or simplification solutions that lead to approximate analysis. Therefore, the physical behaviours of the system such as inductor current, output voltage and physical effects of components will not be accurately predictable. This study presents very accurate equations and all aspects of the structure are predictable. In our research, this issue is investigated in Laplace and Z domains using the output-to-input transfer function calculations, and the effect of converter circuit elements is assessed using equations obtained. For extracting the transfer function, initial values are calculated in the $Z$ domain and based on the final value theorem, converter output voltage and input current have been calculated. Effects of converter components on capacitor voltage and input current ripples have been analysed and reported. In the final step, results of the theoretical analysis were confirmed by simulation results obtained in MATLAB/SIMULINK environment and implementation on a prototype in laboratory scales.
\end{abstract}

Keywords: Components Effects; Continuous and Discontinuous Conduction Modes; DC-DC Power Boost Converter; Output Voltage and Input Current Ripples; Z and Laplace Transform Functions

\section{INTRODUCTION}

DC-DC power boost structure is one of the most important types of converters. This converter increases the input DC voltage to a higher value. Based on the wide use of this converter in industry, renewable energy sources such as photovoltaic panels, hybrid resources, and wind turbines, studying this converter is important in order to get an optimal and stable designing and acceptable limited range of ripples for output voltage and input current and proper efficiency [1], [2]. Having a general and plenary mathematical pattern for a system leads to an accurate prediction of structure behaviors. In literature different mathematical models are investigated, in which impedance mode [3], small signal analysis mode [4]-[6] and steady space average model [7], [8] are the most famous ones. The main disadvantage of the above mentioned models is that they use numerical analysis method or simplification solutions that lead to approximate analysis. For example, based on Impedance models, the order of transfer function will increase for the complex DC-DC power boost converters, so order reduction methods should be used that reduce the accuracy of the results [9].

Another method for the mathematical modeling of the converter is applying the small signal analysis method. In this method, the large signal model of the converter is initially plotted based on the ac and DC small signal analysis. The large signal model can be separated into linearized ac and dc models and by removing the DC components of the model, the equivalent circuit of the ac signal is obtained. Then, using the equivalent ac small signal, the ratio of output parameters of the converter to each of the input parameters is extracted. In this model, to derive the system transfer function, assuming that the converter parameters have small variations around the fixed DC value, it is neglected from the DC part and by drawing the equivalent circuit of the ac small signal, the system transfer function is obtained [10], [11]. If the parameters of the system have great variations around the DC value, then by neglecting from the DC part, the accuracy of the extracted mathematical model will be reduced [12].
The average state space model is another method for modeling the DC-DC converters. By this method, in the first step, the state equations are extracted in both $\mathrm{ON}$ and OFF switching intervals and then the average of each set of equations is obtained by using the duty cycle value, which is a weight coefficient. Finally, two sets of extraction equations are combined with each other and converted into a single equation set [13], [14].

The resulting equation set contains two ac and DC parts. In this method, as in the small signal method, assuming that the converter parameters have small variations, the DC part is neglected and the small ac part is analyzed by the Laplace transform and the transfer function of the system is extracted. So, this method can be useful if the converter parameters have small changes; otherwise, this method would not have the proper accuracy for the analysis of the system. Therefore, the physical behaviors of the system such as inductor current, output voltage and physical effects of components will not be accurately predictable.

The operating modes of the power converters are divided into two: Discontinuous Conduction Mode (DCM) and Continuous Conduction Mode (CCM). In the case of $\mathrm{CCM}$, the input inductor current is always continuous and exits in all time intervals, but in the case of DCM, this value is zero for a time interval and non-zero for another interval. We will analyze converter performance in CCM. It should be considered that the method suggested in this study is also applicable to DCM [15]-[18] and all types of DC-DC Buck and Buck-Boost converters [19], [20].

An exact modeling method should be able to analyze all the operational modes of a system in both transient and steady states. In this study, the object is to present a modeling method based on the mathematics, for analyzing all operational modes of DC-DC power boost converter such as the converter stability, the steady states, and the transient responses. The proposed method is based on two Laplace and $\mathrm{Z}$ transforms. In the proposed method, the Laplace transform is used for obtaining the related relations to the inductor current and the output voltage. The $\mathrm{Z}$ transform is used as a tool for determining the initial values of the inductor current and the output voltage in each 
switching interval. In this research, by applying the presented mathematical model, the effect of the converter components and the load resistance on the electrical parameters of the converter such as output voltage ripple, inductor current ripple, stability, and transient response is analyzed. Finally, the results of the theoretical analysis are compared with the simulation through MATLAB/SIMULINK and PSCAD/EMTDC and experimental results. The proposed method can be a suitable model for designing the circuit elements of the converter and also developing the suitable control methods for controlling the DC-DC converters.

\section{DC-DC BOOST CONVERTER ANALYSIS IN CCM}

A classical boost converter is illustrated in Fig. 1. As can be seen, there is a power switch in the structure, $\mathrm{S}$, that will work in ON and OFF modes.

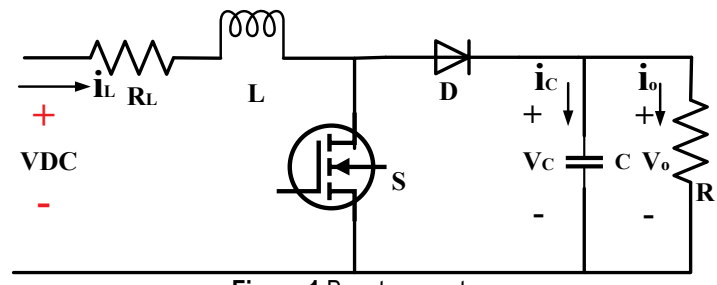

Figure 1 Boost converter

According to Kirchhoff laws, we can write Eq. (1) and (2) based on first order derivations of inductor current and capacitor voltage.

$L \frac{\mathrm{d} i_{L}}{\mathrm{~d} t}+R_{L} i_{L}=f(t) v_{c}-V_{i}$,

$C \frac{\mathrm{d} v_{c}}{\mathrm{~d} t}+\frac{v_{c}}{R}=f(t) i_{L}$,

The most important subject in a mathematical model for a power converter is the power switch modeling. $f(t)$ is the switching function and is modeling the power switch, $S$. This function is used to determine the converter's equations in different time intervals in which the key is being switched on and off as follows:

$f(t)=\sum_{p=0}^{\infty}\left[u\left(t-t_{1}-p T\right)-u(t-T-p T)\right]$,

In this equation, $p$ is switching intervals number and $T$ is period of switching. A switching interval is the total of ON and OFF time. $f(t)$ is the pulses that reach to gatesource or base-emitter pins in order to activate or deactivate the power switches in On and OFF intervals. Fig. 2 shows the $f(t)$ waveform and as can be seen in $\left(0, t_{1}\right)$ time interval, it is equal to zero, so in this condition the power switch will be deactivated and is open circuit in boost converter structure and for $\left(\mathrm{t}_{1}, \mathrm{~T}\right)$ the switch is active and short-circuit. So Eq. (1) and (2) can be written in two different modes according to switch conditions. So $f(t)$ is the total of difference between the two-step functions for $p$ switching intervals.

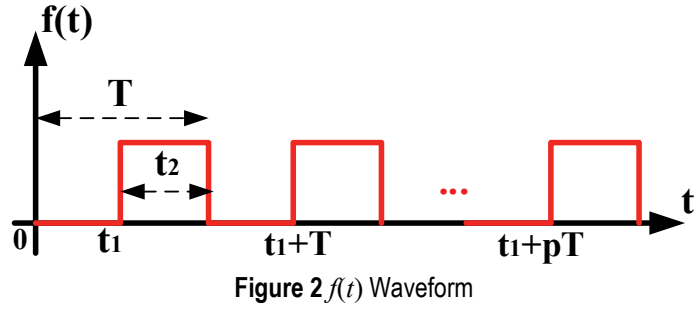

In order to be able to make a mathematical model and do the converter analysis in the discrete time domain, we can apply a variable change by this equation:

$t=(p+q) T$ for $p=0,1,2, L \quad 0 \leq q<1$,

By replacing variable above, $f(t)$ converts to $p$ discrete functions with $q$ variable and we will have a single time system. By replacing this variable change in Eq. (3), $f(t)$ can be written as below:

$$
\begin{aligned}
f(t) & =\sum_{p=0}^{\infty}\left[u\left(p T+q T-t_{1}-p T\right)-u(p T+q T-T-p T)\right]= \\
& =\sum_{p=0}^{\infty}\left[u\left(q T-t_{1}\right)-u(q T-T)\right]=p f(q),
\end{aligned}
$$

In Eq. (5), $f(q)$ will be equal to:

$f(q)=u\left(q T-t_{1}\right)-u(q T-T)$, below:

Also, $u\left(q T-t_{1}\right)$ and $u(q T-T)$ can be calculated as

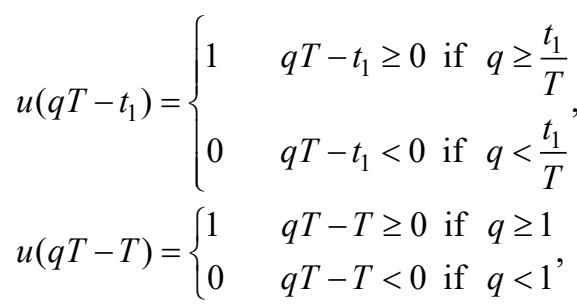

In these equations $D$ is the converter's duty cycle and can be written as:

$D=\frac{t_{1}}{T}$,

By applying this value to Eq. (7), we can rewrite it as:

$f(m)=\left\{\begin{array}{ll}0 & \text { for } 0 \leq m<D \\ 1 & \text { for } D \leq m<1\end{array}\right.$,

$f(q)$ is zero for $[0, D]$, so:

$$
\begin{aligned}
& \frac{\mathrm{d} i_{L, p}}{\mathrm{~d} q}=\frac{T}{L} V_{i}-\frac{T R_{L}}{L} i_{L, p}, \\
& \frac{\mathrm{d} v_{o, p}}{\mathrm{~d} q}=-\frac{T}{R C} v_{o, p},
\end{aligned}
$$


By considering Eq. (10), it can be illustrated that the $[0,1]$ interval can be divided to two $[0, D]$ (when the switch turns $\mathrm{ON}$ ) and $[D, 1]$ (when the switch turns off). Fig. 3 shows the states of the converter when the switch is turned on and off.

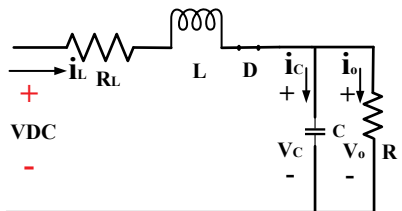

(a)

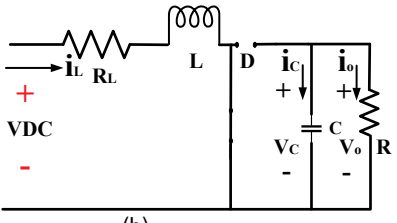

(b)
Figure 3 Equivalent circuit of the boost converter, (a) in $[0, D]$, when the switch is turned on, (b) in $[D, 1]$ when the switch is turned off

So, equations can be rewritten in the following matrix form for the time the switch is on:

$$
\begin{gathered}
{\left[\begin{array}{c}
\frac{\mathrm{d} i_{L, p}}{\mathrm{~d} q} \\
\frac{\mathrm{d} v_{o, p}}{\mathrm{~d} q}
\end{array}\right]=\left[\begin{array}{cc}
-\frac{R_{L} T}{L} & 0 \\
0 & -\frac{T}{R C}
\end{array}\right]\left[\begin{array}{l}
i_{L, p} \\
v_{o, p}
\end{array}\right]+\left[\begin{array}{c}
\frac{T}{L} \\
0
\end{array}\right] V_{i} \text { if } 0 \leq q<D,} \\
i_{L, p}(0)=i_{L 0, p}, v_{o, p}(0)=v_{o 0, p}
\end{gathered}
$$

$i_{L o, p}$ and $v_{o 0, p}$ are initial values of input current and capacitor voltage and in $[0, D]$ interval.

When the switch is turned off, equations for $[D, 1]$ will be:

$$
\begin{aligned}
& \frac{\mathrm{d} i_{L, p}}{\mathrm{~d} q}=\frac{T}{L} V_{i}-\frac{T R_{L}}{L} i_{L, p}-\frac{T}{L} v_{o, p}, \\
& \frac{\mathrm{d} v_{o, p}}{\mathrm{~d} q}=\frac{T}{C} i_{L, p}-\frac{T}{R C} v_{o, p},
\end{aligned}
$$

And these can be written in a matrix form as shown below:

$$
\begin{gathered}
{\left[\begin{array}{c}
\frac{d i_{L, p}}{d q} \\
\frac{d v_{o, p}}{d q}
\end{array}\right]=\left[\begin{array}{cc}
-\frac{R_{L} T}{L} & -\frac{T}{L} \\
\frac{T}{C} & -\frac{T}{R C}
\end{array}\right]\left[\begin{array}{l}
i_{L, p} \\
v_{o, p}
\end{array}\right]+\left[\begin{array}{c}
\frac{T}{L} \\
0
\end{array}\right] \quad \text { if } D \leq q<1,} \\
i_{d, p}(D)=i_{L 1, p} \\
, \quad v_{o, p}(D)=v_{o 1, p}
\end{gathered}
$$

\section{OBTAINING THE INPUT CURRENT AND CAPACITOR VOLTAGE EQUATIONS USING LAPLACE TRANSFORM}

In Eq. (13) and (16), $i_{L 1, p}$ and $v_{o 1, p}$ respectively are primary values of input current and capacitor voltage in $[0$, $D]$ and $[D, 1]$ intervals respectively. By applying LaplaceTransform for these equations, we will have:

$$
\begin{aligned}
& \left(s+\frac{T R_{L}}{L}\right) I_{L, p}(s)=i_{L 0, p}+\frac{T}{L S} V_{i} \quad \text { if } \quad 0 \leq q<D, \\
& \left(s+\frac{T}{R C}\right) V_{o, p}(s)=v_{o 0, p} \text { if } \quad 0 \leq q<D \\
& \left(s+\frac{T R_{L}}{L}\right) I_{L, p}(s)=+i_{L 1, p}+\frac{T}{L S} V_{i}-\frac{T}{L} V_{o, p}(s), D \leq q<1,
\end{aligned}
$$

$$
\left(s+\frac{T}{R C}\right) V_{o, p}(s)=v_{o 1, p}+\frac{T}{C} I_{L, p} \quad \text { if } \quad D \leq q<1,
$$

By sorting Eq. (17) to Eq. (20), in the form of a matrix, the relation between input current and capacitor voltage in the Laplace domain can be written as:

$$
\begin{aligned}
& {\left[\begin{array}{c}
I_{L, p}(s) \\
V_{o, p}(s)
\end{array}\right]=\left[\begin{array}{c}
\frac{1}{s+(\alpha-\gamma) T}\left(i_{L 0, p}+\frac{T}{L} \frac{V_{i}}{s}\right) \\
\frac{v_{o 0, p}}{s+(\alpha+\gamma) T}
\end{array}\right] \text { if } 0 \leq q<D,(21)} \\
& {\left[\begin{array}{c}
I_{L, p}(s) \\
V_{o, p}(s)
\end{array}\right]=\frac{1}{s^{2}+2 \alpha T s+T^{2}\left(\alpha^{2}+\omega^{2}\right)} \times} \\
& \times\left[\begin{array}{c}
(\alpha+\gamma) T+s \quad-\frac{T}{L} \\
\frac{T}{C} \\
s+T(\alpha-\gamma)
\end{array}\right]\left[\begin{array}{c}
i_{L 1, p}+\frac{T V_{i}}{L s} \\
v_{o 1, p}
\end{array}\right] \text { if } D \leq q<1,
\end{aligned}
$$

In these equations, $\alpha, \omega$ and $\gamma$ can be calculated as shown below:

$$
\alpha=\frac{\left(\frac{R_{L}}{L}+\frac{1}{R C}\right)}{2}, \omega=\sqrt{\frac{1}{L C}\left(\frac{R_{L}}{R}+1\right)-\alpha^{2}}, \gamma=\frac{\left(\frac{1}{R C}-\frac{R_{L}}{L}\right)}{2},
$$

By replacing Conversely Laplace function for Eq. (21) and (22), the input current and capacitor voltage will be obtained as follows in the $q$ domain:

$$
i_{L, p}(q)=\left\{\begin{array}{l}
i_{L 0, p} e^{-(\alpha-\gamma) q T}+\frac{V_{i}}{R_{L}}\left[1-e^{-(\alpha-\gamma) m T}\right] \text { if } 0 \leq q<D, \\
\frac{V_{i}}{R_{L}+R}\left[\begin{array}{l}
1-e^{-\alpha(q T-t 1)} \cos \omega\left(q T-t_{1}\right) \\
+\frac{\omega^{2}-\alpha \gamma}{(\gamma+\alpha) \omega} e^{-\alpha\left(q T-t_{1}\right)} \sin \omega\left(q T-t_{1}\right)
\end{array}\right]+ \\
+i_{L 1, p} e^{-\alpha\left(q T-t_{1}\right)}\left[\cos \omega\left(q T-t_{1}\right)+\frac{\gamma}{\omega} \sin \omega\left(q T-t_{1}\right)\right]- \\
-\frac{v_{o 1, p} e^{-\alpha\left(q T-t_{1}\right)}}{\omega L} \sin \omega\left(q T-t_{1}\right) \quad \text { if } \quad D \leq q<1,
\end{array}\right.
$$

$$
v_{o, p}(q)=\left\{\begin{array}{l}
v_{o 0, p} e^{-(\alpha+\gamma) q T} \quad \text { if } \quad 0 \leq q<D, \\
\frac{R V_{i}}{R_{L}+R}\left[\begin{array}{c}
1-e^{-\alpha\left(q T-t_{1}\right)} \cos \omega\left(q T-t_{1}\right) \\
-\frac{\alpha}{\omega} e^{\alpha\left(t_{1}-q T\right)} \sin \omega\left(q T-t_{1}\right)
\end{array}\right]+ \\
+\frac{i_{L 1, p}}{\omega C} e^{-\alpha\left(q T-t_{1}\right)} \sin \omega\left(q T-t_{1}\right)+ \\
+v_{o 1, p} e^{\alpha\left(t_{1}-q T\right)}\left[\begin{array}{c}
\cos \omega\left(q T-t_{1}\right)+ \\
\frac{\gamma}{\omega} \sin \omega\left(t_{1}-q T\right)
\end{array}\right] \text { if } \quad D \leq q<1,
\end{array}\right.
$$

In Eq. (26) and (27), $i_{L o, p}, v_{o 0, p}, i_{L 1, p}$ and $v_{o 1, p}$ are unknown parameters and for obtaining in a simpler state, the number of unknown values should be reduced. For this issue, we can calculate $i_{L 1, p}$, and $v_{o 1, p}$ in terms of $i_{L o, p}$ and $v_{o 0, p}$. By considering continuity of functions $i_{L}(t)$ and $v_{o}(t)$, we can write: 
$\lim _{t \rightarrow\left(t_{1}+p T\right)^{+}} i_{L}(t)=\lim _{t \rightarrow\left(t_{1}+p T\right)^{+}} i_{L}(t)$

Eq. (25) in terms of $m$ can be written as:

$$
\lim _{q \rightarrow(D)^{-}} i_{L, p}(q)=\lim _{q \rightarrow(D)^{+}} i_{L, p}(q)
$$

By replacing relation (24) into (27), $i_{L 1, p}$ can be obtained in terms of $i_{L 0, p}$ in the following way:

$i_{L 1, p}=i_{L 0, p} e^{-(\alpha-\gamma) t_{1}}+\frac{V_{i}}{R_{L}}\left[1-e^{-(\alpha-\gamma) t_{1}}\right]$

For output voltage we can also write:

$$
\begin{aligned}
\lim _{t \rightarrow\left(t_{1}+p T\right)^{-}} v_{o}(t) & =\lim _{t \rightarrow\left(t_{1}+p T\right)^{+}} v_{o}(t), \\
\lim _{q \rightarrow(D)^{-}} v_{o, p}(q) & =\lim _{q \rightarrow(D)^{+}} v_{o, p}(q),
\end{aligned}
$$

And, $v_{o 1, p}$ based on $v_{o 0, p}$ can be calculated as:

$v_{o 1, p}=v_{o 0, p} e^{-(\alpha-\gamma) t_{1}}$,

In the final step, by replacing Eq. (28) and (31) in Eq. (24) and (25), the relationship between input current and capacitor voltage can be calculated in the following way:

$$
\begin{aligned}
& \int i_{L 0, p} e^{-(\alpha-\gamma) q T}-\frac{V_{i}}{R_{L}}\left[e^{-(\alpha-\gamma) q T}-1\right] \text { if } 0 \leq q<D, \\
& i_{L, p}(q)=\left\{\begin{array}{l}
\frac{V_{i}}{R_{L}+R}\left[\begin{array}{l}
1-e^{-\alpha\left(q T-t_{1}\right)} \cos \omega\left(q T-t_{1}\right)- \\
\frac{\omega^{2}-\alpha \gamma}{\omega(\alpha+\gamma)} e^{-\alpha\left(q T-t_{1}\right)} \sin \omega\left(t_{1}-q T\right)
\end{array}\right]+ \\
+\left[\left(i_{L 0, p}-\frac{V_{i}}{R_{L}}\right) e^{\gamma t_{1}-\alpha q T}+\frac{V_{i} e^{-\alpha\left(q T-t_{1}\right)}}{R_{L}}\right] \times \\
\times\left[\cos \omega\left(q T-t_{1}\right)-\frac{\gamma}{\omega} \sin \omega\left(t_{1}-q T\right)\right]+
\end{array}\right. \\
& +\frac{v_{o 0, p} e^{-\gamma t_{1}-\alpha q T}}{\omega L} \sin \omega\left(t_{1}-q T\right) \quad \text { if } \quad D \leq q<1,
\end{aligned}
$$

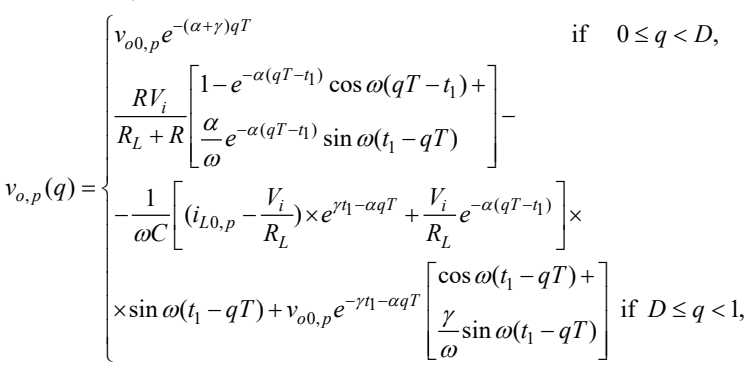

\section{PRIMARY VALUES CALCULATIONS FOR INDUCTOR CURRENT AND CAPACITOR VOLTAGE USING Z FUNCTION}

Due to the continuity condition of the input current and capacitor voltage, these equations are always true in $t=p T$ : $\lim _{t \rightarrow(p T)^{-}} i_{L}(t)=\lim _{t \rightarrow(p T)^{+}} i_{L}(t)$

This equation can be used in terms of $q$ as:

$\lim _{q \rightarrow 1^{-}} i_{L, p}(m)=\lim _{q \rightarrow 0^{+}} i_{L, p+1}(q)$

For capacitor voltage, we will have:

$\lim _{t \rightarrow(p T)^{-}} v_{o}(t)=\lim _{t \rightarrow(p T)^{+}} v_{o}(t)$

This equation can also be written in terms of $q$ as:

$\lim _{q \rightarrow 1^{-}} v_{o, p}(q)=\lim _{q \rightarrow 0^{+}} v_{o, p+1}(q)$

By applying $t_{2}=T-t_{1}$, the following relationships are calculated for the initial values of the input current and output voltage in the $p+1$ interval:

$i_{L 0, p+1}=\frac{V_{i}}{R_{L}+R}\left[\begin{array}{l}1-e^{-\alpha t_{2}} \cos \omega t_{2} \\ -e^{-\alpha t_{2}} \frac{\alpha \gamma-\omega^{2}}{(\alpha+\gamma) \omega} \sin \omega t_{2}\end{array}\right]+$
$+\left[\left(i_{L 0, p}-\frac{V_{i}}{R_{L}}\right) e^{\gamma t_{1}-\alpha T}+\frac{V_{i} e^{-\alpha t_{2}}}{R_{L}}\right] \times$

$\times\left(\frac{\gamma}{\omega} \sin \omega t_{2}+\cos \omega t_{2}\right)-\frac{v_{o 0, p}}{\omega L} \sin \omega t_{2} e^{-\gamma t_{1}-\alpha T}$,

$v_{o 0, p+1}=\frac{R V_{i}}{R_{L}+R}\left[\sin \omega t_{2}\left(1-e^{-\alpha t_{2}} \cos \omega t_{2}-\frac{\alpha}{\omega} e^{-\alpha t_{2}}\right)\right]+$

$+\frac{\sin \omega t_{2}}{\omega C}\left[\left(i_{L 0, p}-\frac{V_{i}}{R_{L}}\right) e^{\gamma t_{1}-\alpha T}+\frac{V_{i} e^{-\alpha t_{2}}}{R_{L}}\right]-$

$-v_{o 0, p} e^{-\gamma t_{1}-\alpha T}\left(\frac{\gamma}{\omega} \sin \omega t_{2}-\cos \omega t_{2}\right)$,

Eq. (38) and (39) are two differential equations. To solve this kind of equations, we can use $Z$ transform. To a discrete time function, the following relationships can establish:

$\mathrm{Z}\left\{i_{L 0, p}\right\}=I_{L 0}(z), \mathrm{Z}\left\{v_{0, p}\right\}=V_{0}(z)$

Using the time priority property of $Z$ transform, Eq. (41), always is true for $i_{L 0, n+1}$ and $v_{o 0, n+1}$ :

$$
\begin{aligned}
& x[p+1] \stackrel{Z \text { domain }}{\longleftrightarrow} z X(z)-z x(0) \Rightarrow \\
& Z\left\{i_{L 0, p+1}\right\}=z I_{L 0}(z)-z i_{L 0,0}, \\
& Z\left\{v_{0, p+1}\right\}=z V_{0}(z)-z v_{0,0},
\end{aligned}
$$

$i_{L \mathrm{o}, 0}$ and $v_{o 0,0}$ are initial values of inductor current and capacitor voltage in $t=0$. By applying $Z$-transform in relations (38) and (39) and writing equations in the form of matrices, according to Eq. (42) the equations $I_{L O}(Z)$ and $V_{o O}(z)$ can establish: 
$[X] \times\left[\begin{array}{c}I_{L 0}(z) \\ V_{0}(z)\end{array}\right]=\left[\begin{array}{c}\frac{z}{z-1} b_{1}+z i_{L 0,0} \\ \frac{z}{z-1} b_{2}+z v_{0,0}\end{array}\right]$,

In this equation $X, b_{1}$ and $b_{2}$ can be gained as shown below:

$X=\left[\begin{array}{cc}z-e^{-\alpha T+\gamma t_{1}}\left(\begin{array}{l}\cos \omega t_{2} \\ +\frac{\gamma}{\omega} \sin \omega t_{2}\end{array}\right) & \frac{e^{-\alpha T-\gamma t_{1}} \sin \omega t_{2}}{L \omega} \\ -\frac{e^{-\alpha T-\gamma t_{1}} \sin \omega t_{2}}{\omega C} & z-e^{-\alpha T-\gamma t_{1}}\left(\cos \omega t_{2}-\frac{\gamma}{\omega} \sin \omega t_{2}\right)\end{array}\right]$,

$b_{1}=\left\{\frac{R_{L}}{R_{L}+R}\left[\sin \omega t_{2} \frac{\omega^{2}-\alpha \gamma}{\omega(\alpha+\gamma)} e^{-\alpha t_{2}}-e^{-\alpha t_{2}} \cos \omega t_{2}+1\right]+\right.$

$\left.+\left(e^{-\alpha t_{2}}-e^{\gamma t_{1}-\alpha T}\right)\left(\frac{\gamma}{\omega} \sin \omega t_{2}+\cos \omega t_{2}\right)\right\} \frac{V_{i}}{R_{L}}$,

$b_{2}=\left[\begin{array}{l}-\frac{R}{R_{L}+R}\left(e^{-\alpha t_{2}} \cos \omega t_{2}+\frac{\alpha}{\omega} e^{-\alpha t_{2}} \sin \omega t_{2}+1\right)+ \\ +\frac{1}{\omega R_{L} C}\left(-e^{\gamma t_{1}-\alpha T}+e^{-\alpha t_{2}}\right) \sin \omega t_{2}\end{array}\right] V_{i}$,

By solving Eq. (42), $I_{L O}(Z)$ and $V_{o O}(z)$ values will be equal to:

$\left[\begin{array}{l}I_{L 0}(z) \\ V_{o 0}(z)\end{array}\right]=X^{-1} \times\left[\begin{array}{c}z I_{L 0,0}+b_{1} \frac{z}{z-1} \\ z V_{o 0,0}+b_{2} \frac{z}{z-1}\end{array}\right]$,

In this equation, the value of $X^{-1}$ can be obtained as:

$X^{-1}=\frac{1}{|X|} \times\left[\begin{array}{cc}z-\left(\begin{array}{cc}-\frac{\gamma}{\omega} \sin \omega t_{2}+ \\ +\cos \omega t_{2}\end{array}\right) e^{-\alpha T-\gamma t_{1}} & -\frac{e^{-\alpha T-\gamma t_{1}} \sin \omega t_{2}}{\omega L} \\ \frac{e^{-\alpha T+\gamma t_{1}} \sin \omega t_{2}}{\omega C} & z-e^{-\alpha T+\gamma t_{1}}\left(\begin{array}{l}\cos \omega t_{2} \\ +\frac{\gamma}{\omega} \sin \omega t_{2}\end{array}\right)\end{array}\right]$,

And $|X|$ is:

$|X|=e^{-2 \alpha T}-2 z e^{-\alpha T} \cos \phi T+z^{2}$,

$\cos \phi T=\frac{\gamma}{\omega} \sin \omega t_{2} \sinh \gamma t_{1}+\cos \omega t_{2} \cosh \gamma t_{1}$,

By replacing $X^{-1}$ in Eq. (45), $I_{L O}(Z)$ and $V_{o O}(z)$ can be calculated as:

$$
\begin{aligned}
& I_{L 0}(z)=\frac{z}{z^{2}-2 z e^{-\alpha T} \cos \phi T+e^{-2 \alpha T}} \times \\
& \times\left\{\left[-e^{-\alpha T-\gamma t_{1}}+z\left(\cos \omega t_{2}-\frac{\gamma}{\omega} \sin \omega t_{2}\right)\right] i_{L 0,0}-\frac{v_{o 0,0} e^{-\alpha T-\gamma t_{1}} \sin \omega t_{2}}{\omega L}+\right. \\
& \left.+\left\{\left[z-e^{-\alpha T-\gamma t_{1}}\left(\cos \omega t_{2}-\frac{\gamma}{\omega} \sin \omega t_{2}\right)\right] b_{1}-\frac{b_{2} e^{-\alpha T-\gamma t_{1}} \sin \omega t_{2}}{\omega L}\right\} \frac{1}{z-1}\right\},
\end{aligned}
$$

$$
\begin{aligned}
& V_{o 0}(z)=\frac{z}{z^{2}-2 z e^{-\alpha T} \cos \phi T+e^{-2 \alpha T}} \times \\
& \times\left\{\left[-e^{-\alpha T+\gamma t_{1}}+z\left(\frac{\gamma}{\omega} \sin \omega t_{2}+\cos \omega t_{2}\right)\right] v_{o 0,0}+\right. \\
& +\frac{i_{L 0,0} e^{-\alpha T+\gamma t_{1}} \sin \omega t_{2}}{\omega C}+\left\{\frac{e^{-\alpha T+\gamma t_{1}} \sin \omega t_{2}}{\omega C} b_{1}+\right. \\
& \left.\left.+\left[z-e^{-\alpha T+\gamma t_{1}}\left(\cos \omega t_{2}+\frac{\gamma}{\omega} \sin \omega t_{2}\right)\right] b_{2}\right\} \frac{1}{z-1}\right\},
\end{aligned}
$$

In order to obtain the initial values of capacitor voltage and input current in a steady state, we can use the final value theorem in $Z$ domain.

$i_{L 0, s s}=\lim _{Z \rightarrow 1}(z-1) I_{L 0}(z)$,
$v_{o 0, s s}=\lim _{Z \rightarrow 1}(z-1) V_{o 0}(z)$,

So, these values can be obtained in a steady state by:

$$
\begin{aligned}
& i_{L 0, s s}= \frac{b_{1}\left[-e^{-\alpha T-\gamma t_{1}}\left(\cos \omega t_{2}-\frac{\gamma}{\omega} \sin \omega t_{2}\right)+1\right]-b_{2} \frac{e^{-\alpha T-\gamma t_{1}} \sin \omega t_{2}}{L \omega}}{e^{-2 \alpha T}+-2 e^{-\alpha T} \cos \phi T+1}, \\
& v_{o 0, s s}=\frac{b_{1} \frac{e^{-\alpha T+\gamma t_{1}} \sin \omega t_{2}}{\omega C}+b_{2}\left[z-e^{-\alpha T+\gamma t_{1}}\left(\cos \omega t_{2}+\frac{\gamma}{\omega} \sin \omega t_{2}\right)\right]}{e^{-2 \alpha T}-2 e^{-\alpha T} \cos \phi T+1},
\end{aligned}
$$

By defining Eq. (53a) and Eq. (53b), one can write the relationship (49) and (50) in terms of steady-state values. By summarizing Eq. (49) and Eq. (50) using relations (53a) and $(53 \mathrm{~b})$ and extending them and using the partial fraction method, Eq. (54a) and Eq. (54b) are obtained as follows:

$$
\begin{aligned}
& I_{d 0}(z)=\frac{z\left[z-e^{-\alpha T} \cos (\phi T)+k e^{-\alpha T}\right] i_{d 0,0}}{z^{2}-2 z e^{-\alpha T} \cos (\phi T)+e^{-2 \alpha T}}- \\
& -\frac{z e^{-\alpha T-\gamma t_{1}} \sin \omega t_{2}}{z^{2}-2 z e^{-\alpha T} \cos (\phi T)+e^{-2 \alpha T}} \frac{v_{0,0}}{\omega L}+ \\
& +\frac{z i_{d 0,0}}{z-1}-\frac{z\left[i_{d 0 s s} z-2 i_{d 0 s s} e^{-\alpha T} \cos (\phi T)+i_{d 0 s s}-b_{1}\right]}{z^{2}-2 z e^{-\alpha T} \cos (\phi T)+e^{-2 \alpha T}}, \\
& V_{0}(z)=\frac{z e^{-\alpha T-\gamma t_{1}} \sin \omega t_{2}}{z^{2}-2 z e^{-\alpha T} \cos (\phi T)+e^{-2 \alpha T}} \frac{i_{d 0,0}}{\omega C}+ \\
& +\frac{z\left[z-e^{-\alpha T} \cos (\phi T)-k e^{-\alpha T}\right] v_{0,0}}{z^{2}-2 z e^{-\alpha T} \cos (\phi T)+e^{-2 \alpha T}}+ \\
& +\frac{z v_{0 s s}}{z-1}-\frac{z\left[v_{0 s s} z-2 v_{0 s s} e^{-\alpha T} \cos (\phi T)+v_{0 s s}-b_{2}\right]}{z^{2}-2 z e^{-\alpha T} \cos (\phi T)+e^{-2 \alpha T}},
\end{aligned}
$$

Using a Reverse $Z$ transform, values of the initial input current and output voltage are obtained in the time domain. To find inverse relations of Eq. (54a) and Eq. (54b), two coupling relations of $Z$ transform are used as follows: 
$\frac{1-\left[r \cos \omega_{0}\right] z^{-1}}{1-\left[2 r \cos \omega_{0}\right] z^{-1}+r^{2} z^{-2}} \stackrel{Z}{\longleftrightarrow}\left[r^{n} \cos \omega_{0} p\right] u[p]$,

$\frac{\left[r \sin \omega_{0}\right] z^{-1}}{1-\left[2 r \cos \omega_{0}\right] z^{-1}+r^{2} z^{-2}} \stackrel{Z}{\longleftrightarrow}\left[r^{n} \sin \omega_{0} p\right] u[p]$,

Using Eq. (55a) and separating the fractions of Eq. (53a) and (53b) and writing them in the form of two equations based on Eq. (54), relations between the initial conditions of the input current and output voltage in the discrete time domain can be gained as Eq. (56) and Eq. (57):

$$
\begin{aligned}
& i_{d 0, p}=i_{d 0 s s}+\left(i_{d 0,0}-i_{d 0 s s}\right) e^{-\alpha p T} \cos (\phi p T)+ \\
& +\frac{e^{-\alpha p T} \sin (\phi p T)}{\sin (\phi T)}\left[\begin{array}{l}
k i_{d 0,0}-\frac{e^{-\gamma t_{1}} \sin \omega t_{2}}{\omega L} v_{0,0} \\
+\frac{i_{d o s s} e^{-\alpha T} \cos (\phi T)-i_{d 0 s s}+b_{1}}{e^{-\alpha T}}
\end{array}\right], \\
& v_{0, p}=v_{0 s s}+\left(v_{0,0}-v_{0 s s}\right) e^{-\alpha p T} \cos (\phi p T)+ \\
& +\frac{e^{-\alpha p T} \sin (\phi p T)}{\sin (\phi T)}\left[\begin{array}{l}
\frac{e^{-\gamma t_{1}} \sin \omega t_{2}}{\omega L} v_{0,0}-k v_{0,0} \\
+\frac{v_{o s s} e^{-\alpha T} \cos (\phi T)-v_{0 s s}+b_{2}}{e^{-\alpha T}}
\end{array}\right],
\end{aligned}
$$

\section{INDUCTOR CURRENT AND CAPACITOR VOLTAGE RIPPLES ANALYSIS}

Initial values of input current $i_{L 0, s s}$ and capacitor voltage $v_{o 0, s s}$ in steady-state can be obtained by using the final value of $Z$ transform as follows:

$$
\begin{aligned}
& v_{o 0, s s}=\frac{b_{1} \frac{e^{-\alpha T+\gamma t_{1}} \sin \omega t_{2}}{\omega C}+b_{2}\left[z-e^{-\alpha T+\gamma t_{1}}\left(\frac{\gamma}{\omega} \sin \omega t_{2}+\cos \omega t_{2}\right)\right]}{e^{-2 \alpha T}-2 e^{-\alpha T} \cos \phi T+1}, \\
& i_{L 0, s s}=\frac{b_{1}\left[1-e^{-\alpha T-\gamma t_{1}}\left(-\frac{\gamma}{\omega} \sin \omega t_{2}+\cos \omega t_{2}\right)\right]-b_{2} \frac{e^{-\alpha T-\gamma t_{1}} \sin \omega t_{2}}{\omega L}}{e^{-2 \alpha T}-2 e^{-\alpha T} \cos \phi T+1},
\end{aligned}
$$

Fig. 4 illustrates the average values of inductor and capacitor voltages and currents in a steady-state situation.

To obtain the value of the capacitor voltage, we can use the following equation:

$V_{o}=\frac{1}{T} \int_{t^{\prime}}^{t^{\prime}+T} v_{o}(t) \mathrm{d} t$

In this equation, $t^{\prime}$ is any desired moment of time.

By applying the change of the variable presented in Eq.

(4) in Eq. (60), the result can be written as:

$V_{o}=\int_{0}^{1} v_{o, s s}(q) \mathrm{d} q$,
In the above relation $v_{o 0, s s}(q)$ is the output voltage value in a steady-state condition, which is obtained by replacing Eq. (29) in Eq. (32):

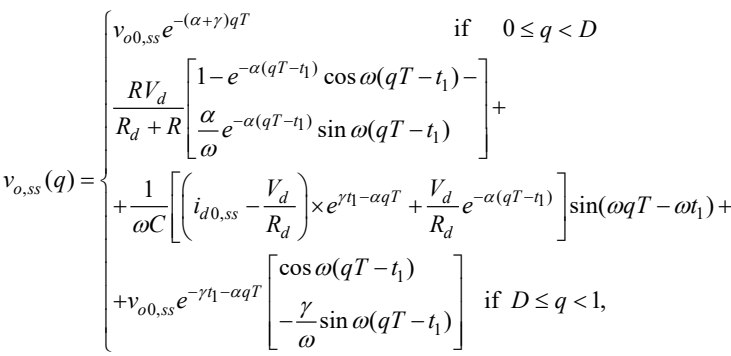

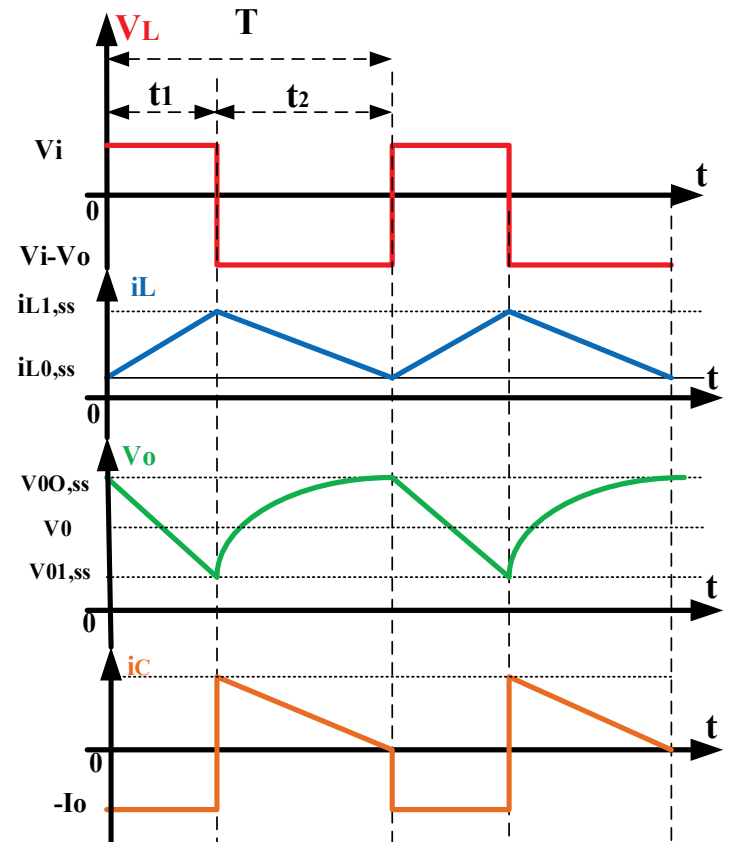

Figure 4 Input voltage and current, output current and voltage waveforms of Boost converter in CCM

By replacing Eq. (62) into Eq. (61) in one switching interval, the mean value of the capacitor voltage can be calculated as shown in equation below:

$$
\begin{aligned}
& V_{o}=\frac{v_{o 0, s s}}{(\alpha+\gamma) T}\left(1-e^{-(\alpha+\gamma) D T}\right)+u_{1}\left[1-a_{0}\left(a_{1}-\frac{\alpha}{\omega} a_{2}\right)-2 \alpha a_{3}-D\right]+ \\
& +\frac{i_{L 1, s s}}{\omega C}\left(a_{0} a_{2}+\omega a_{3}\right)+v_{o 1, s s}\left[a_{0}\left(a_{1}-\frac{\gamma}{\omega} a_{2}\right)-a_{3}(\gamma-\alpha)\right],
\end{aligned}
$$

In Eq. (63) the values of $u_{1}, a_{0}$ until $a_{3}$ can be illustrated as follows:

$u_{1}=\frac{R V_{i}}{R_{L}+R}$

$a_{0}=\frac{e^{-\alpha t_{2}}}{\left(\alpha^{2}+\omega^{2}\right) T^{2}}$,

$a_{1}=T\left(\omega \cos \omega t_{2}-\alpha \sin \omega t_{2}\right)$,

$a_{2}=-T\left(\omega \sin \omega t_{2}+\alpha \cos \omega t_{2}\right)$,

$a_{3}=\frac{1}{\left(\alpha^{2}+\omega^{2}\right) T}$ 


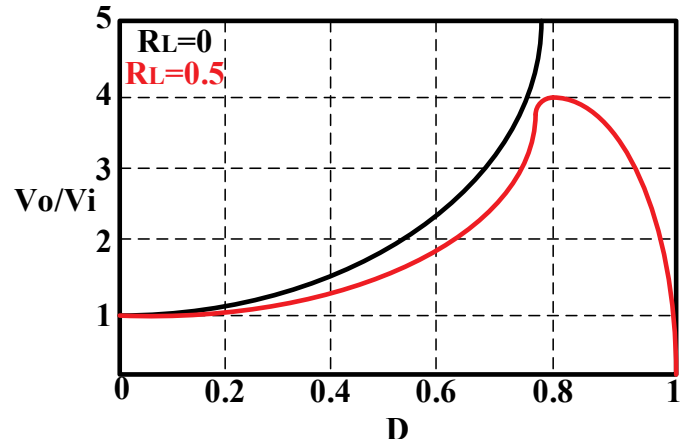

Figure $\mathbf{5}$ Variation curve of average capacitor voltage to input voltage ratio in terms of duty cycles for a boost converter in Continuous Conduction Mode

Fig. 5 shows the variation curve of capacitor voltage's mean value to input voltage ratio in a steady-state mode in terms of duty cycle. This curve is obtained for two lossless $\left(R_{L}=0\right)$ and with loss $\left(R_{L} \neq 0\right)$ states by considering $V_{i}=$ $10 \mathrm{VDC}, R=35 \Omega, C=0.33 \mathrm{mF}, L=4 \mathrm{mH}$, and $f=10$ $\mathrm{kHz}$. The figure shows that the average value of capacitor voltage increases by increasing the duty cycle. As can be seen, for larger duty cycles, the lossless curve is different from lossy state, and in this case with larger values of duty cycles, the average value of the capacitor voltage decreases and for $D=1$, this value is equal to zero.

In Fig. $5 I_{o}$ is the average value of inductor current in steady-state mode. To calculate the value of this parameter, like the output voltage, the following equation can be used:

$I_{L}=\int_{0}^{1} i_{L, s S}(q) \mathrm{d} q$

In the above relation $i_{L, s s}(q)$ is the output voltage value in steady-state, which is obtained by replacing Eq. (34) in (31):

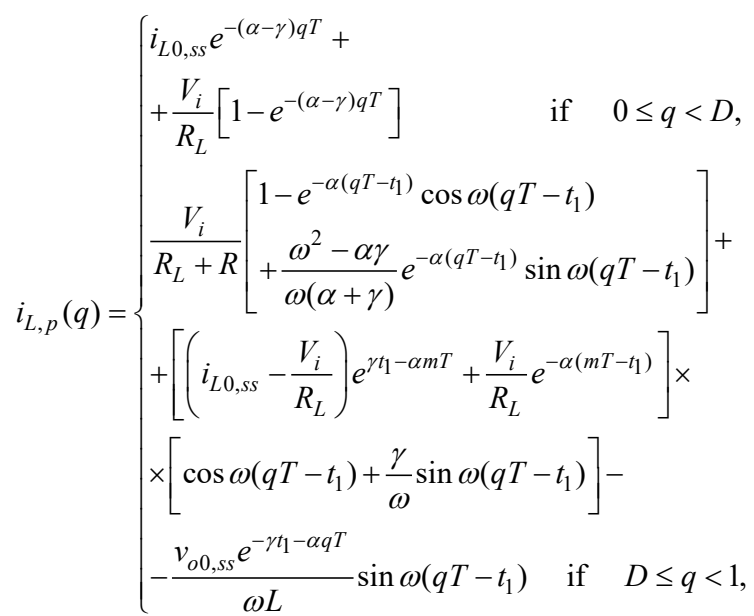

By calculating the integral value in one switching interval, the mean value of input current can be obtained as:

$$
\begin{aligned}
& I_{o}=\frac{i_{L 0, s s}}{(\alpha-\gamma) T}\left[1-e^{-(\alpha-\gamma) t_{1}}\right]+\frac{V_{i}}{R_{L}}\left[D+\frac{1}{(\alpha-\gamma) T}\left(e^{-(\alpha-\gamma) t_{1}}-1\right)\right]+ \\
& +i_{L 1, s s}\left[a_{0}\left(a_{1}+\frac{\gamma}{\omega} a_{2}\right)-a_{3}(\gamma-\alpha)\right]-\frac{v_{o 1, s s}}{\omega L}\left(a_{0} a_{2}+\omega a_{3}\right)+ \\
& +u_{2}\left[1-a_{0}\left(a_{1}-u_{3} a_{2}\right)+a_{3}\left(\omega u_{3}-\alpha\right)-D\right]
\end{aligned}
$$

In this equation, $u_{2}$ and $u_{3}$ can be obtained by:

$u_{2}=\frac{V_{i}}{R_{L}+R}, \quad u_{3}=\frac{\omega^{2}-\alpha \gamma}{\omega(\alpha+\gamma)}$,

One of the influential parameters in the performance of converters which should be considered in the designing is the value of capacitor voltage ripple. Therefore, the circuit components should be designed in a way that output has a minimum value of ripple. In this section, this parameter is calculated and the effect of each circuit component and duty cycle on capacitor voltage ripple is investigated. With respect to Fig. 4, it can be seen that the output voltage ripple in CCM will be equal to:

$\Delta V_{o}=v_{o 0, s s}-v_{o 1, s s}$,

In this equation, $v_{o 0, s s}$ is the maximum output voltage and can be written as:

$v_{o 0, s s}=\frac{\frac{e^{-\alpha T+\gamma t_{1}} \sin \omega t_{2}}{C \omega} b_{1}+\left[z-e^{-\alpha T+\gamma t_{1}}\left(\cos \omega t_{2}+\frac{\gamma}{\omega} \sin \omega t_{2}\right)\right] b_{2}}{e^{-2 \alpha T}-2 e^{-\alpha T} \cos \phi T+1}$

And $v_{o 1, s s}$ is the minimum value of capacitor voltage ripple in steady-state and will be obtained as below:

$v_{o 1, s s}=\frac{\frac{e^{-\alpha T+\gamma t_{1}} \sin \omega t_{2}}{\omega C} b_{1}+\left[z-e^{-\alpha T+\gamma t_{1}}\left(\cos \omega t_{2}+\frac{\gamma}{\omega} \sin \omega t_{2}\right)\right] b_{2}}{e^{-2 \alpha T}-2 e^{-\alpha T} \cos \phi T+1} \times$

$x e^{-(\alpha+\gamma) t_{1}}$,

So by replacing this Eq. in (73), output voltage ripple can be calculated as:

$$
\begin{aligned}
& \Delta V_{o, s s}=\frac{\frac{e^{-\alpha T+\gamma t_{1}} \sin \omega t_{2}}{\omega C} b_{1}+\left[z-e^{-\alpha T+\gamma t_{1}}\left(\cos \omega t_{2}+\frac{\gamma}{\omega} \sin \omega t_{2}\right)\right] b_{2}}{e^{-2 \alpha T}-2 e^{-\alpha T} \cos \phi T+1} \times \\
& \times\left(1-e^{-(\alpha+\gamma) t_{1}}\right),
\end{aligned}
$$

Another effective parameter in the performance of converters is the inductance current. Given that during the switch-on-and-off moments, the inductor current passes through the switch and the load current is charged through the inductor, calculation of the input current ripple and evaluation of the effect of converter elements on this value is one of the most important issues. By considering Fig. 5, it can be seen that the value of the input current ripple in the steady-state situation can be calculated from the difference between the minimum and maximum values of the inductor current:

$\Delta I_{L, s s}=i_{L 1, s s}-i_{L 0, s s}$,

In this equation, $i_{L 0, s s}$ and $i_{L 1, s s}$ are minimum and maximum values of the input current in steady-state mode respectively. Thus, it can be calculated as: 


$$
i_{L 1, s s}=i_{L 0, n} e^{-(\alpha-\gamma) t_{1}}+\frac{V_{i}}{R_{L}}\left[1-e^{-(\alpha-\gamma) t_{1}}\right],
$$

So, the value of the current ripple in the inductor will be equal to:

$$
\begin{aligned}
& \Delta I_{L S s}=-\frac{\left[1-e^{-\alpha T-\gamma t_{1}}\left(\cos \omega t_{2}-\frac{\gamma}{\omega} \sin \omega t_{2}\right)\right] b_{1}-\frac{e^{-\alpha T-\gamma t_{1}} \sin \omega t_{2}}{\omega L} b_{2}}{e^{-2 \alpha T}-2 e^{-\alpha T} \cos \phi T+1} \times \\
& \times\left(1-e^{-(\alpha-\gamma) t_{1}}\right)+\frac{V_{i}}{R_{L}}\left[1-e^{-(\alpha-\gamma) t_{1}}\right],
\end{aligned}
$$

\section{CIRCUITS COMPONENTS EFFECT ON CAPACITOR'S VOLTAGE AND INDUCTOR'S CURRENT}

To make theoretical analysis, the curves have been obtained based on the current of the inductor, output voltage, and their ripples. In order to plot output voltage ripple curves in terms of converter parameters, the values of converter elements should be chosen in a way that converter stays in CCM.

In the DC-DC boost converter, the division of working modes can be done based on parameters such as input voltage, duty cycle, and value of the converter's load resistance. Given the small amount of $R_{L}$, we can neglect the amount of this resistance in our calculations and by using the critical inductance relationship presented in [21], the boundary between two modes of CCM and DCM is determined:

$L_{C}=\frac{R}{2 f} D(1-D)^{2}$,

In this equation, $L_{C}$ is the critical value of the inductance between two continuous and discontinuous conduction working modes. This means that DC-DC boost converter for $L<L_{C}$ will be in $D C M$ case and will stay in $\mathrm{CCM}$ for $L>L_{C}$. To perform theoretical analysis, converter elements are chosen as follows: $V_{i}=10-15 V D C, R=10$ $50 \Omega, f=10 \mathrm{kHz}, R_{L}=0.5 \Omega, C=0.33 \mathrm{mF}$ and $D=0.68$. Regarding the selected values, using the Eq. (80), the critical inductance value between two modes of CCM and DCM will be equal to $L=1.2 \mathrm{mH}$. To perform theoretical analysis, the value is considered as $L=6 \mathrm{mH}$, due to the fact that this value is larger than its critical, the converter will be in the DCM. Capacity voltage and input current ripples in steady-state mode are shown as a percentage of the mean value of the capacitor voltage and input current in terms of load resistance, inductance, capacitance and converter duty cycles in Fig. 6, 7 and 8.

Fig. 6 shows the capacitor voltage and input current ripples in terms of load resistance and different duty cycles. As shown in Fig. 6a, for a heavy amount of loads, where current is high and the resistance value is small, output voltage ripple is maximum. This value is also reduced by increasing the load resistance. As can be seen, for a given value of load resistance, as the amount of duty cycle increases, the value of the output voltage ripple also increases. As shown in Fig. 6b, unlike the output voltage ripples, the value of the inductor current ripple for high load resistance is maximum and with an increase in load resistance, the value of ripple of inductor current also increases. According to the figure, it can be seen that the converter duty cycle has a nonlinear relation with the value of the inductor current and for a given value of load resistance, the value of the inductor current ripple rises as the duty cycle increases to a specified value, which is between 0.2 and 0.4 , and after that as the duty cycles of converter increase, the amount of ripple in inductor current decreases.

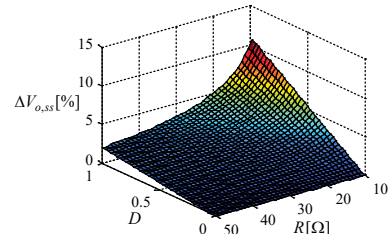

(a)

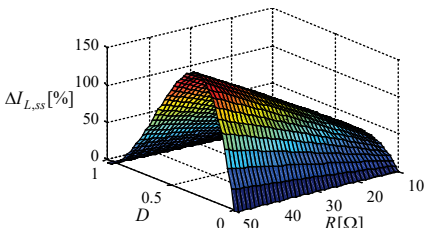

(b)
Figure 6 Change percentage curve for (a) capacitor voltage ripple; (b) input current ripple, in terms of load and duty cycle

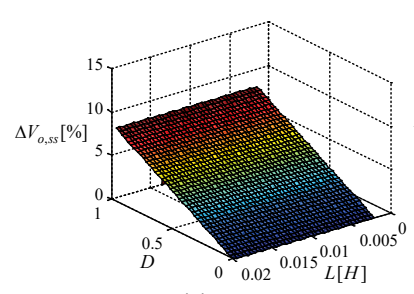

(a)

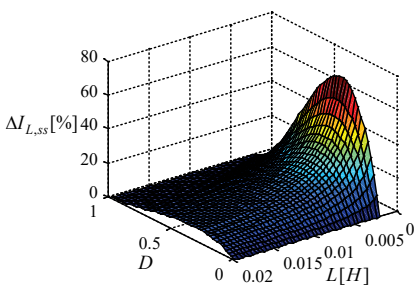

(b)
Figure 7 Change percentage curve for (a) output voltage ripple; (b) inductor current ripple, in terms of inductor value and duty cycle

Fig. 7 shows the effect of inductance and duty cycle of the converter on capacitor voltage and input current ripples. As shown in Fig. 7a, the output voltage is independent of inductance in CCM, so the voltage remains constant by changing the value of inductance. But, according to Fig. $7 \mathrm{~b}$, the amount of inductance affects the amount of input current ripple, and with a change in inductance, the value of the ripple also changes and the inductance current decreases as the inductance increases.

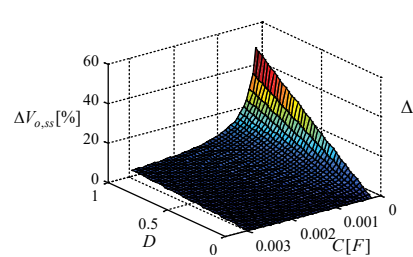

(a)

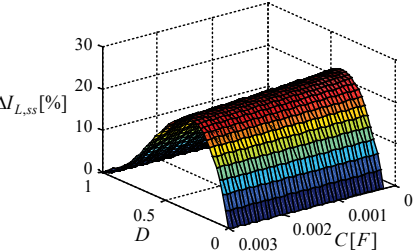

(b)
Figure 8 Change percentage curve for (a) output voltage ripple; (b) inductor current ripple, in terms of capacitance and duty cycle

Fig. 8 illustrates the effect of the capacitance and duty cycle of the converter on capacitor voltage and input current ripples. The effect of the capacitance on values of the output voltage and the current of the inductor is in contrast to the effect of inductance. Contrary to the inductance, this does not affect the output voltage ripples. The change in the capacitance causes changes in the value of capacitor voltage ripples and by increasing the capacitance value, voltage ripples decrease. It can be seen in detail in Fig. $8 \mathrm{~b}$ that the capacitance does not affect the amount of the input current ripple. The effect of the duty cycle on the output voltage ripple is linear and this effect is significant only in small values of capacitances. 


\section{EXPERIMENTAL RESULTS AND DISCUSSION}

To evaluate the performance of the proposed structure and accuracy of the theoretical analysis, a laboratory prototype is implemented. Tab. 1 presents the value of the parameters in our simulation and Tab. 2 illustrates the components values in the prototype.

Table 1 Main parameters in simulation

\begin{tabular}{|c|c|}
\hline Parameter & Value \\
\hline Vin & $10 \mathrm{VDC}$ \\
\hline Capacitor & $1 \mathrm{mf}$ and $1.6 \mathrm{mF}$ \\
\hline Inductor & $4 \mathrm{mH}$ and $15 \mathrm{mH}$ \\
\hline Duty Cycle & 0.68 \\
\hline Power MOSFET & IRFP450 \\
\hline Power Diode & MUR880 \\
\hline Frequency & $10 \mathrm{kHz}$ \\
\hline$R_{\text {LOAD }}$ & 10 and $60 \Omega$ \\
\hline
\end{tabular}

Table 2 Main parameters of implemented prototype

\begin{tabular}{|c|c|}
\hline Parameter & Value \\
\hline Vin & $10 \mathrm{VDC}$ \\
\hline Capacitor & $0.33 \mathrm{mF}$ \\
\hline Inductor & $4 \mathrm{mH}$ \\
\hline Duty Cycle & 0.5 \\
\hline Power MOSFET & IRFP450 \\
\hline Power Diode & MUR880 \\
\hline Frequency & $10 \mathrm{kHz}$ \\
\hline$R_{\text {LOAD }}$ & 50 and $100 \Omega$ \\
\hline
\end{tabular}

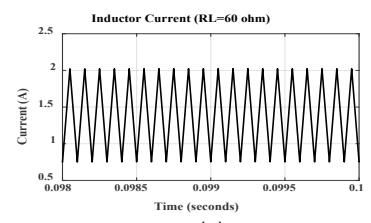

(a)

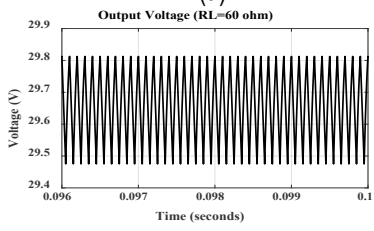

(c)

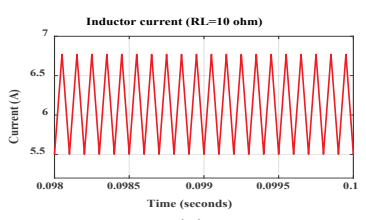

(b)

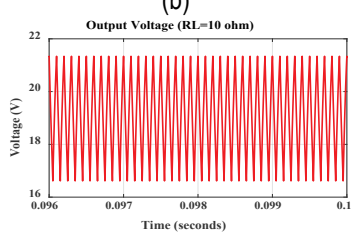

(d)
Figure 9 Inductor current ripples when (a): $R=10 \Omega$, (b): $60 \Omega$, and output voltage ripples when (c) $R=10 \Omega$ and (d): $R=60 \Omega$

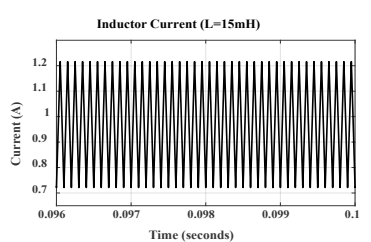

(a)

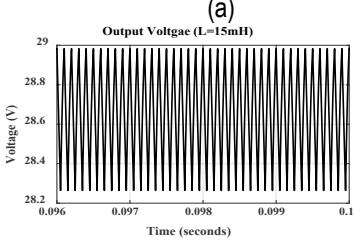

(c)

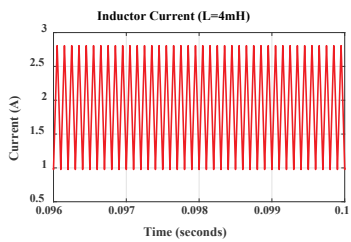

(b)

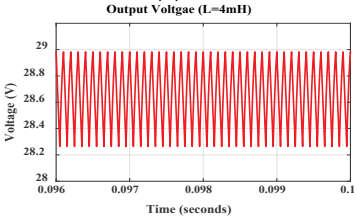

(d)
Figure 10 Inductor current and capacitor voltage changes when inductor changes

As can be seen in Fig. 9, for a higher amount of loads inductor current ripple increases and output voltage decreases. Also, simulation results in an incensement for capacitor voltage ripple for a lower amount of load while we can see a decrement for inductor current ripple. Fig. 9a and $9 \mathrm{~b}$ illustrate inductor current change and Fig. 9c and $9 \mathrm{~d}$ show the capacitor voltage ripples.

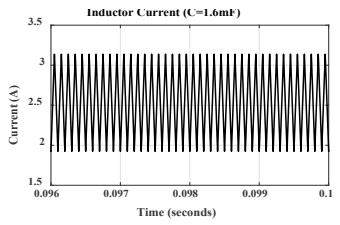

(a)

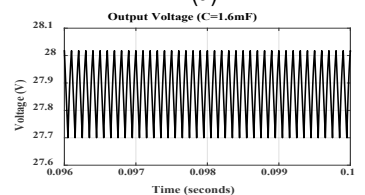

(c)

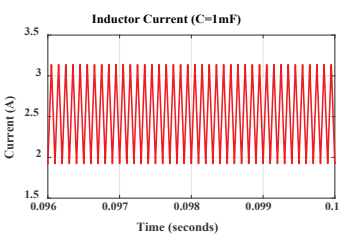

(b)

(d)

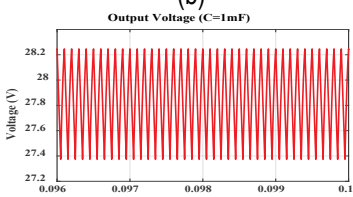

Figure 11(a) and (b), Inductor current ripples are fixed for different values of capacitors, but capacitor voltage ripples are changing. This ripple is smaller for

(c) higher values of the capacitor and higher for (d) smaller capacitors.

Fig. 10 illustrates the input current and output voltage ripples for different values of the inductor. As we expected, higher values of ripples are reported for small inductances and output voltage ripple is completely independent inductance.

The same condition can be seen for different capacitance values in Fig. 11 and by changing in the amount of capacitance value, only output ripples are changing and for this ripple the value is smaller for higher values of the capacitor. So, simulation results completely confirm our theoretical results.

Fig. 12 illustrates our implemented prototype results. 19.8 VDC for output voltage has obtained for a $50 \Omega$ resistive load and 20.1 VDC for $100 \Omega$ while current value is $0.82 \mathrm{~A}$ and $0.41 \mathrm{~A}$ for $50 \Omega$ and $100 \Omega$ output load respectively. As the figure illustrates, all experimental results are confirming the theoretical and simulation outcomes with a high reliability.

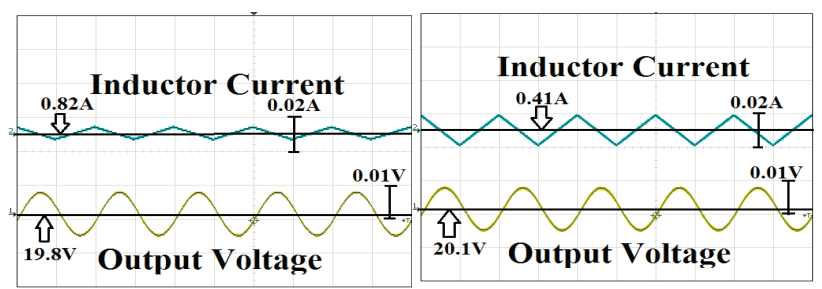

(a)

(b)

Figure 12 Inductor current and capacitor voltage waveforms when (a) $R_{\mathrm{LOAD}}=$ $50 \Omega$ and (b) $R_{\mathrm{LOAD}}=100 \Omega$

\section{CONCLUSION}

In different references, by assuming the small changes for DC components of power boost converter, only the ac small signal part is analyzed, therefore the results cannot be accurate and with higher changes in DC values, all of the calculations cannot be valid and especially input inductor current and output capacitor voltage and ripples values are not predictable.

Unlike all existing models, our proposed method is more comprehensive and accurate, thus it can help us to evaluate all specifications and behaviors of the circuit. Our study is based on two Laplace and $Z$ transforms. In converter modeling, Laplace transform is considered to determine the inductor current and the capacitor voltage equations, and $Z$ transform was applied as a tool for finding the initial values of these current and voltage signals. It was 
also shown that $\mathrm{Z}$ transform is applicable for transient and permanent state responses analysis. The effects of DC-DC converter elements on electrical parameters such as output voltage and inductor current ripples were analyzed mathematically and it has been proved that output voltage ripple value is in a direct relation with the amount of load and duty cycle, but inductor current is depending directly on load value but it has a nonlinear relation with duty cycle value and for 0.5 of duty cycle current ripple value is the maximum. Also, results show that inductor current and capacitor voltage ripples have no connection with capacitor and inductor values respectively while voltage directly increases for small values of capacitors and decreases for higher amounts of capacitors and current ripples is high for small inductor and is less for higher inductor values.

A group of simulations based on theoretical analysis has been done by MATLAB/SIMULINK and PSCAD/EMTDC and also a laboratory scaled prototype has been implemented and results confirming all mathematical calculations. Results indicate that the value output voltage is more for higher values of load. For example, for $50 \Omega$ of load, we reached $19.8 \mathrm{VDC}$ while for $100 \Omega, 20.1$ VDC has been gained.

\section{REFERENCES}

[1] Wu, H., Sun, K., Chen, R., Hu, H., \& Xing, Y. (2012). Fullbridge three-port converters with wide input voltage range for renewable power systems. IEEE Transactions on Power Electronics, 29(7), 3965-3974. https://10.1109/TPEL.2012.2188105

[2] Hu, Y., Xiao, W., Cao, W., Ji, B., \& Morrow, J. (2015). Three-port DC-DC converter for stand-alone photovoltaic systems. IEEE Transactions on Power Electronics, 30(6), 3068-3076. https://10.1109/TPEL.2014.2331343

[3] Kim, D., Son, D., \& Choi, B. (2006). Input impedance analysis of PWM DC-to-DC converters. Twenty-First Annual IEEE Applied Power Electronics Conference and Exposition, 2006. APEC '06. 1339-1346. https://10.1109/APEC.2006.1620713

[4] Luo, F. L. \& Ye, H. (2007). Small signal analysis of energy factor and mathematical modeling for power dc-dc converters. IEEE Transactions on Power Electronics, 22(1), 69-79. https://10.1109/TPEL.2006.886652

[5] Barry, B. C., Hayes, J. G., \& Rylko, M. S. (2015). CCM and DCM operation of the interleaved two-phase boost converter with discrete and coupled inductors. IEEE Transaction on Power Electronics, 30(12), 6551-6567. https://10.1109/TPEL.2014.2386778

[6] Wang, Q., Shi, L., \& Chang, C. (2008). Small-signal transfer functions for a single-switch buck-boost converter in continuous conduction mode. $9^{\text {th }}$ International Conference on Solid-State and Integrated-Circuit Technology, 20162019. https://10.1109/ICSICT.2008.4734960

[7] Siwakoti, Y. P. \& Blaabjerg, F. (2017). Single Switch Nonisolated Ultra-StepUp DC-DC Converter With an Integrated Coupled Inductor for High Boost Applications. IEEE Transactions on Power Electronics, 32(11), 85448558.

[8] Barry, B. C., Hayes, J. G., Rylko, M. S., Stala, R., Penczek, A., Mondzik, A., \& Ryan, R. T. (2017). Digital type II compensation with forced-output control of an interleaved two-phase coupled-inductor boost converter. IEEE Energy Conversion Congress and Exposition (ECCE), Cincinnati, OH, 4935-4941. https://10.1109/ECCE.2017.8096836

[9] Haroun, R., El-Aroudi, A., Pastor, A.C., Garcia, G., Olalla, C., \& Salamero, L. M. (2015). Impedance Matching in Photovoltaic Systems Using Cascaded Boost Converters and
Sliding-Mode Control. IEEE Transactions on Power Electronics, 30(6), 3185-3199. https://10.1109/TPEL.2014.2339134

[10] Gajanayake, C. J., Vilathgamuwa, D. M., \& Loh, P. C. (2005). Small-signal and signal-flow-graph modeling of switched Z-source impedance network. IEEE Power Electronics Letters, 3(3), 111-116. https://10.1109/LPEL.2005.859771

[11] Liu, S., Liu, P. X., \& Wang, X. (2016). Stochastic SmallSignal Stability Analysis of Grid-Connected Photovoltaic Systems. IEEE Transactions on Industrial Electronics, 63(2), 1027-1038. https://10.1109/TIE.2015.2481359

[12] Hamad, W., Wanckel, S., \& Hofmann, W. H. E. (2016). Small-Signal Analysis of Ultra-High-Speed Multi-Mode VCSELs. IEEE Journal of Quantum Electronics, 52(7), 2400311. https://10.1109/JQE.2016.2574540

[13] Klumpner, C., Ponggorn, K., \& Rashed, M. (2013). Modelling and control of a multistage interleaved DC-DC converter with coupled inductors for the super-capacitor energy storage system. IET Power Electronics, 6(7), 13601375. https://10.1049/iet-pel.2012.0529

[14] Liu, H. \& Li, H. F. (2015). Novel high step-up DC-DC converter with the active coupled-inductor network for a sustainable energy system. IEEE Transactions on Power Electronics, 30(12), 6476-6482, 2015. https://10.1109/TPEL.2015.2429651

[15] Cruz, J. M. L., Saldierna, L. H. D., \& Ramos, L. H. L. (2013). Switching regulator using a high step-up voltage converter for fuel-cell modules. IET Power Electronics, 6(8), 16261633. https://10.1049/iet-pel.2012.0433

[16] Lai, C. M., Lin, Y. C., \& Lee, D. (2015). Study and implementation of a two-phase interleaved bidirectional DC/DC converter for vehicle and DC-microgrid systems. Energies, 8, 9969-9991. https://10.3390/en8099969

[17] Zhang, W., Xu, D., Li, X., Xie, R., Li, H., Dong, D., Sun, C., $\&$ Chen, M. (2013). Seamless transfer control strategy for the fuel cell uninterruptible power supply system. IEEE Trans. Power Electron, 28(2), 717-729. https://10.1109/TPEL.2012.2204777

[18] Sun, X., Shen, Y., Zhu, Y., \& Guo, X. (2015). Interleaved boost-integrated LLC resonant converter with fixedfrequency PWM control for renewable energy generation applications. IEEE Transactions on Power Electronics, 30(8), 4312-4326. https://10.1109/TPEL.2014.2358453

[19] Kececioglu, O. F., Gani, A., Acikgoz, H., \& Sekkeli. M. (2018). Design of LQR controller based on particle swarm optimization for optimal control of DC-DC buck converter. $3^{r d}$ International Mediterranean Science and Engineering Congress, 843-847.

[20] Gani, A., Kececioglu, O. F., Acikgoz, H., \& Sekkeli, M. (2018). Dynamic performance comparison of LQR and LQI controllers on buck converter. International Congress on Engineering and Architecture.

[21] Kazimierczuk, M. K. (2008) Pulse-Width Modulated DCDC Power Converters. UK, Wiley.

\section{Contact information:}

Davood GHADERI, Ph.D., Assist. Professor (Corresponding author)

Electrical and Electronics Engineering Department,

Faculty of Engineering and Natural Sciences,

Bursa Technical University, Bursa, Turkey

E-mail: davood.ghaderi@btu.edu.tr

Gökay BAYRAK, PhD, Assist. Professor

Electrical and Electronics Engineering Department,

Faculty of Engineering and Natural Sciences,

Bursa Technical University, Bursa, Turkey

E-mail: gokay.bayrak@btu.edu.tr 